\title{
Relation between DNA ionization potentials, single base substitutions and pathogenic variants
}

\author{
Fabrizio Pucci ${ }^{1,2}$ and Marianne Rooman ${ }^{1 *}$
}

From Varl-COSI 2018: identification and annotation of genetic variants in the context of structure, function, and disease Chicago, IL, USA. 08 July 2018

\begin{abstract}
Background: It is nowadays clear that single base substitutions that occur in the human genome, of which some lead to pathogenic conditions, are non-random and influenced by their flanking nucleobase sequences. However, despite recent progress, the understanding of these "non-local" effects is still far from being achieved.

Results: To advance this problem, we analyzed the relationship between the base mutability in specific gene regions and the electron hole transport along the DNA base stacks, as it is one of the mechanisms that have been suggested to contribute to these effects. More precisely, we studied the connection between the normalized frequency of single base substitutions and the vertical ionization potential of the base and its flanking sequence, estimated using MP2/6-31G* ab initio quantum chemistry calculations. We found a statistically significant overall anticorrelation between these two quantities: the lower the vIP value, the more probable the substitution. Moreover, the slope of the regression lines varies. It is larger for introns than for exons and untranslated regions, and for synonymous than for missense substitutions. Interestingly, the correlation appears to be more pronounced when considering the flanking sequence of the substituted base in the $3^{\prime}$ rather than in the $5^{\prime}$ direction, which corresponds to the preferred direction of charge migration. A weaker but still statistically significant correlation is found between the ionization potentials and the pathogenicity of the base substitutions. Moreover, pathogenicity is also preferentially associated with larger changes in ionization potentials upon base substitution.
\end{abstract}

Conclusions: With this analysis we gained new insights into the complex biophysical mechanisms that are at the basis of mutagenesis and pathogenicity, and supported the role of electron-hole transport in these matters.

Keywords: Single base substitutions, Pathogenic mutations, Charge transport, DNA base stacking, Vertical ionization potential

\section{Background}

The understanding of the biophysical mechanisms that drive single DNA base substitutions in different regions of the genome is one of the key questions of the postgenomic era. Indeed, the effects of these mutation processes are potentially responsible for a range of diseases such as cancer and various neurodevelopment disorders.

\footnotetext{
*Correspondence: mrooman@ulb.ac.be

${ }^{1}$ Computational Biology and Bioinformatics, Université Libre de Bruxelles, Roosevelt Ave. 50 1050, Bruxelles, Belgium

Full list of author information is available at the end of the article
}

The rationalization of these mechanisms is very complex, since single base substitutions (SBSs) can be triggered by a wide range of factors, e.g. chemical species, physical agents or enzymes, through mechanisms such as base deamination, base depurination, or tautomeric shifts. These effects moreover depend on the nucleotide sequence context $[1,2]$.

The analysis of the large quantity of available genomic data has allowed to firmly state that SBSs do not occur randomly along the genome. For example, one of the wellknown mutational signatures observed in cancer genomes 
occurs at mCpG dinucleotides [3, 4]. Methylated cytosines $(\mathrm{mC})$ undergo more frequently spontaneous deamination than unmethylated cytosines, which leads to the $\mathrm{C} \rightarrow \mathrm{T}$ transition with higher probability. The second signature, which has been observed in different cancer types, occurs at $\mathrm{TpC}$ dinucleotides in which the cytosine is mutated through transition or transversion. It has been related to the overactivity of the APOBEC cytidine deaminases $[5,6]$. Other signatures are specific to certain types of cancers or diseases [7-9]. For example, the C:G $\rightarrow$ A:T transversion is a well-known mutation in lung cancer induced by tobacco carcinogens. The "UV signature", namely $\mathrm{C} \rightarrow \mathrm{T}$ mutations at dipyrimidine sites, is caused by ultraviolet light via cyclobutane pyrimidine dimer formation and is commonly found in melanomas.

It became recently clear that there is an important effect of the flanking base sequence on SBSs [2], which appears to extend beyond the dinucleotide units [10]. However, the mechanisms triggering these "non-local" effects are far from clear. Their comprehension is of primary importance and would lead to deep insights into mutagenesis phenomena and the deleteriousness of genetic variations.

In this context, the physical phenomena of electron-hole transfer along the DNA stack comes into play. Exposure to high-energy radiations or to reactive oxygen species generated as by-products of the cellular metabolism can lead to DNA ionization through the creation of electron holes. These holes then migrate along the DNA stack until they remain (more or less) localized in a potential well [11-13].

Although some electron holes have solely a damaging effect and need to be repaired by specific enzymes [14], others seem to have a positive role as suggested more than a decade ago by the finding that the amount of reactive oxygen species likely to create such holes is regulated by specific proteins and is, for example, higher during cell differentiation [15]. The important role played by electron-hole transfer in various biological processes appears increasingly clear $[16,17]$ and more specifically, in the sequence dependence of the SBSs $[2,12,13,18]$ and their possible pathogenicity $[12,19]$.

Important steps towards the understanding of longdistance hole transfer in DNA have been achieved. However, large parts of the picture are still lacking. Indeed, experimental measures are challenging due to the complexity of working at the nanoscale [20,21], and accurate quantum chemistry calculations are extremely heavy for these systems [22, 23].

In this paper, we further analyzed the relationship between the electron-hole transfer along the DNA stack, the sequence-dependence of the mutability and the pathogenicity of the mutations. For that purpose, we calculated the vertical ionization potential (vIP) of all short nucleobase sequences and correlate these to the presence of benign and/or deleterious mutations occurring in cancer and inherited disorders, with the goal of confirming the prominent contribution of hole migration in these matters.

\section{Results}

\section{Mutation frequencies in the SBS dataset}

We have set up a dataset of single base substitutions which occur in genes, i.e. in exons, introns or untranslated regions (UTRs), and are annotated as pathogenic, benign, of unclear significance, etc, as described in Methods. In exons, we distinguish between synonymous and missense variants.

Let us start by analyzing which of the base pairs and which of the dinucleotide or trinucleotide stacks (also called doublets and triplets) mutate significantly more or less frequently than expected from random substitutions in the different gene regions. Note that the triplets that we consider are defined as three successive bases and usually do not correspond to codons, even if located in exons. The results are summarized in Table 1, and reported exhaustively in Additional file 2: Table S4.

First, the well-known bias towards mutations that occur at C:G base pairs rather than at A:T [18] is clearly observed for missense, synonymous, UTR and intron mutations. Indeed, the frequency of mutations of $\mathrm{C}$ or $\mathrm{G}$ is 1.3 to 1.5 higher than expected on the basis of

Table 1 Normalized SBS frequencies, defined as the ratio between the frequencies of mutations in the nucleobase motifs and the observation frequencies of the same motifs in exons (with a distinction between missense and synonymous SBSs), introns and UTRS

\begin{tabular}{|c|c|c|c|c|c|c|c|}
\hline Missense & & Synonymous & & Intron & & UTR & \\
\hline $\mathrm{G}: \mathrm{C}$ & 1.27 & $\mathrm{G}: \mathrm{C}$ & 1.39 & $\mathrm{G}: \mathrm{C}$ & 1.53 & $\mathrm{G}: \mathrm{C}$ & 1.31 \\
\hline $\mathbf{C p G}$ & 3.33 & $\mathbf{C p G}$ & 5.40 & $\mathbf{C p G}$ & 8.70 & $\mathbf{C p G}$ & 4.10 \\
\hline $\mathbf{G p G}$ & 1.74 & $\mathbf{G p G}$ & 2.75 & $\mathbf{G p G}$ & 1.97 & $\mathbf{G p G}$ & 1.69 \\
\hline CGT & 4.87 & $\mathrm{ACG}$ & 6.62 & $C \mathbf{G C}$ & 12.80 & CGT & 5.58 \\
\hline$C \mathbf{G G}$ & 4.35 & $C \mathbf{C G}$ & 6.54 & $C \mathbf{G G}$ & 12.48 & $\mathrm{ACG}$ & 4.81 \\
\hline $\mathrm{CCG}$ & 4.21 & $C \mathbf{G G}$ & 6.20 & $C \mathbf{C G}$ & 11.70 & $C \mathbf{C G}$ & 4.67 \\
\hline$A: T$ & 0.70 & $A: T$ & 0.57 & $A: T$ & 0.64 & $A: T$ & 0.71 \\
\hline $\mathbf{A p G}$ & 0.54 & $\mathbf{T p C}$ & 0.40 & $\mathbf{A p A}$ & 0.53 & $\mathbf{T p G}$ & 0.59 \\
\hline ApA & 0.50 & $\mathbf{A p C}$ & 0.39 & $\mathbf{T p A}$ & 0.51 & $\mathbf{T p C}$ & 0.49 \\
\hline $\mathrm{CAA}$ & 0.44 & $\mathrm{GAT}$ & 0.24 & $C \mathbf{T} A$ & 0.40 & $\mathrm{CAA}$ & 0.43 \\
\hline $\mathrm{T} \mathbf{T G}$ & 0.33 & $\mathrm{GAC}$ & 0.19 & $C \mathbf{A A}$ & 0.37 & $\lceil\mathbf{T} C$ & 0.39 \\
\hline $\mathrm{GAA}$ & 0.30 & $\mathrm{GAA}$ & 0.15 & $\mathrm{G} \mathbf{A} A$ & 0.32 & $\mathrm{GAA}$ & 0.33 \\
\hline $\mathrm{R}_{\text {All }}$ & 2.0 & $R_{\text {All }}$ & 4.7 & RAll & 2.0 & $\mathrm{R}_{\text {All }}$ & 2.0 \\
\hline $\mathrm{R}_{\mathrm{CpG}}$ & 4.9 & $R_{C p G}$ & 9.4 & $R_{C p G}$ & 4.5 & $\mathrm{R}_{\mathrm{CpG}}$ & 3.3 \\
\hline
\end{tabular}

The nucleotide at which the substitution occurs is in bold. The mutations that are more frequent than expected are shown in the upper section of the Table, those that are less frequent in the middle section, and the ratio of transitions to transversions (noted $\mathrm{R}$ ) in the lower section. Exhaustive results are given in Additional file 2: Table S4 
the frequency of $\mathrm{C}: \mathrm{G}$ pairs in the corresponding region, whereas SBSs at $\mathrm{A}$ or $\mathrm{T}$ are 0.6 to 0.7 times less frequent than expected. Note that C:G pairs are slightly underrepresented in the human genome (around 40\%) but not in the human exome (around 50-55\%).

The preference for SBSs to occur on the $\mathrm{C}$ base of $\mathrm{CpG}$ dinucleotides is even more pronounced, with a rate that is 3 to 9 times higher than expected from the CpG frequency. $\mathrm{CpGs}$ are underrepresented in the whole genome, which can be attributed to the fact that they are the preferential target of DNA-methyltransferase: this enzyme methylates the cytosine that can more easily undergo the base substitution $\mathrm{mCpG} \rightarrow \mathrm{TpG}[18,24]$.

The other doublet that is preferentially targeted by mutations is GpG (with the substituted base in bold). Its mutation rate is from 2 to 3 times higher than expected. In contrast, the least mutated dinucleotides are $\mathbf{T p N}$ and ApN where $\mathrm{N}=\mathrm{A}, \mathrm{C}$ or $\mathrm{G}$, with a rate that is 6 to 18 times lower than the $\mathrm{CpG}$ mutation rate.

Among triplets, SBSs occur preferentially at the middle base of CGG, CCG, CGT, CGC and ACG, in all regions (some are just below the threshold, see Additional file 2: Table S4). At the other extreme, GAA and some other triplets with A- or T-base substitutions occur at rates that are up to 40 times lower than the most frequent SBS triplets.

Interestingly, the observed trends are almost identical for missense and UTR mutations, and are amplified for synonymous and intronic mutations: the most frequently mutated motifs are even more frequent, and the least frequent SBSs are even less frequent. This amplification is quite strong: for $\mathrm{CpG}$ doublets, for example, the frequency of SBSs is 9 times higher in introns than expected from the frequency of this doublet, 5 times higher for synonymous SBSs, and only 3-4 times for UTR and missense SBSs. For GAA triplets, which are less frequent than expected, the frequency ratio is 0.3 for missense, UTR and intron mutations, and only 0.15 for synonymous mutations. The reason of these differences is currently unclear, as mentioned in the "Discussion" section.

Finally, we observed that the majority of SBSs are transitions. Indeed, the ratio of transitions to transversions is equal to 2 in the missense, intron and UTR subgroups. These values are close to those already observed in other analyses $[25,26]$. However this ratio increases up to 5 for the synonymous mutations. Moreover, it substantially increases at CpG sites where it reaches values between 3 and 9.

\section{Relation between the vIP of nucleobase motifs and the SBS}

\section{frequencies}

An important biophysical mechanism that can be related to the sequence dependence of the SBSs is the presence and migration of radical cations (electron holes) along the
DNA molecules. Indeed, due to oxidative stress caused by physical or chemical agents, an electron can be extracted from the DNA. The electron hole then starts migrating along the aromatic rings of the stacked nucleobases, until it remains trapped in a minimum of the ionization potential. The hole can be filled by specific DNA repair proteins or, if not, trigger a base substitution.

In order to quantify these long-range effects and their relation with SBSs, we started by calculating the vIP of all 4 nucleobases, 16 nucleobase doublets, 64 triplets and 256 quadruplets using second-order Møller-Plesset perturbation theory as described in Methods. These vIP values, which are directly related to the probability of extraction of an electron from the DNA base-stacking structure, are given in Additional file 2: Table S4. We also computed the vIP of all nucleobase quintuplets by taking the mean of the vIPs of the two overlapping quadruplets, and of the sextuplets as the mean of the three overlapping quadruplets. For the single-base vIPs, we used the experimental values given in Table 2, which are slightly different from the calculated ones and yield better results in the present context, as expected; note that no experimental values are available for polynucleotide stacks.

We computed the linear and non-linear correlation coefficients between the vIP of the wild type nucleotide motifs and the frequency of observation of the motifs in the SBS dataset normalized by the frequency of their occurrences in the corresponding gene regions (see Methods). These correlations were calculated separately for synonymous and missense mutations in exons, for mutations in introns and UTRs, and for different nucleobase motif lengths, i.e. doublets $(\mathrm{XN})$, triplets $(\mathrm{NXN})$, quadruplets (NXNN), quintuplets (NNXNN) and sextuplets $(\mathrm{NNXNNN})$, where $\mathrm{X}$ indicates the position of the nucleobase substitution in the sequence motif, and $\mathrm{N}$ any of the four bases. The results together with statistical significance tests are shown in Table 3, Additional file 1: Table S1-S3, Additional file 2: Table S4, Fig. 1 and Additional file 1: Figure S1-S4.

Let us first focus on the general behavior displayed by SBSs. When all possible substitutions are considered together, there is a clear, statistically significant, effect of the frequency modulation of the SBSs by the vIP of the wild-type nucleobase and its neighboring sequence. This "non-local" effect is already visible at the dinucleotide level, and clearly extends to longer sequence motifs. For doublets, the linear correlation coefficient between normalized SBS frequencies and vIP values is between -0.4 and -0.5 . This correlation smoothly decreases when

Table 2 Experimental vIP values of nucleotide bases (eV) $[48,49]$

\begin{tabular}{llll}
\hline Gua & Ade & Cyt & Thy \\
\hline $8.20( \pm 0.1)$ & $8.42( \pm 0.1)$ & $8.85( \pm 0.1)$ & $9.10( \pm 0.1)$
\end{tabular}


Table 3 Pearson's linear correlation coefficient between the vIP of the wild-type nucleotide motifs and their normalized SBS frequency in the different gene regions

\begin{tabular}{|c|c|c|c|c|c|}
\hline$x$ & DoubletsXN & Triplets $N X N$ & Quadruplets NXNN & QuintupletsNNXNN & Sextuplets NNXNNN \\
\hline \multicolumn{6}{|c|}{ Missense mutations in exons } \\
\hline All & -0.39 & $\underline{-0.39}(<0.05)$ & $\underline{-0.35}(<0.01)$ & $\underline{-0.34}\left(<10^{-13}\right)$ & $\underline{-0.31}\left(<10^{-51}\right)$ \\
\hline G & -0.23 & $\underline{-0.54}(<0.05)$ & $\underline{-0.36}(<0.01)$ & $\underline{-0.32}\left(<10^{-6}\right)$ & $\underline{-0.32}\left(<10^{-13}\right)$ \\
\hline C & -0.81 & $\underline{-0.71}(<0.005)$ & $\underline{-0.59}\left(<10^{-5}\right)$ & $\underline{-0.54}\left(<10^{-14}\right)$ & $\underline{-0.48}\left(<10^{-56}\right)$ \\
\hline A & 0.77 & $\underline{0.55}(<0.05)$ & $\underline{0.39}(<0.001)$ & $\underline{0.36}\left(<10^{-6}\right)$ & $\underline{0.23}\left(<10^{-17}\right)$ \\
\hline $\mathrm{T}$ & -0.06 & 0.18 & -0.05 & -0.03 & -0.07 \\
\hline \multicolumn{6}{|c|}{ Synonymous mutations in exons } \\
\hline All & -0.48 & $\underline{-0.42}(<0.005)$ & $\underline{-0.39}\left(<10^{-8}\right)$ & $\underline{-0.38}\left(<10^{-29}\right)$ & $\underline{-0.34}\left(<10^{-109}\right)$ \\
\hline G & -0.65 & $\underline{-0.67}(<0.005)$ & $\underline{-0.50}(<0.00005)$ & $\underline{-0.42}\left(<10^{-14}\right)$ & $\underline{-0.33}\left(<10^{-32}\right)$ \\
\hline C & -0.80 & $\underline{-0.68}(<0.05)$ & $\underline{-0.54}(<0.005)$ & $\underline{-0.53}\left(<10^{-13}\right)$ & $\underline{-0.50}\left(<10^{-50}\right)$ \\
\hline A & -0.59 & 0.02 & -0.09 & 0.00 & -0.05 \\
\hline $\mathrm{T}$ & -0.87 & 0.00 & -0.21 & $\underline{-0.17}(<0.05)$ & $\underline{-0.13}(<0.0005)$ \\
\hline \multicolumn{6}{|c|}{ Mutations in introns } \\
\hline All & -0.39 & $\underline{-0.42}(<0.005)$ & $\underline{-0.38}\left(<10^{-5}\right)$ & $\underline{-0.36}\left(<10^{-24}\right)$ & $\underline{-0.33}\left(<10^{-78}\right)$ \\
\hline G & -0.27 & $\underline{-0.51}(<0.05)$ & $\underline{-0.36}(<0.05)$ & $\underline{-0.31}\left(<10^{-6}\right)$ & $\underline{-0.23}\left(<10^{-11}\right)$ \\
\hline C & -0.78 & $\underline{-0.70}(<0.05)$ & $\underline{-0.61}\left(<10^{-7}\right)$ & $\underline{-0.55}\left(<10^{-20}\right)$ & $\underline{-0.54}\left(<10^{-83}\right)$ \\
\hline A & 0.80 & $\underline{0.67}(<0.05)$ & $\underline{0.45}(<0.01)$ & $\underline{0.22}(<0.005)$ & $\underline{0.09}\left(<10^{-8}\right)$ \\
\hline $\mathrm{T}$ & 0.07 & 0.41 & $\underline{0.34}(<0.005)$ & -0.09 & -0.11 \\
\hline \multicolumn{6}{|c|}{ Mutations in UTRs } \\
\hline All & -0.43 & $\underline{-0.39}(<0.05)$ & $\underline{-0.35}(<0.005)$ & $\underline{-0.34}\left(<10^{-16}\right)$ & $\underline{-0.32}\left(<10^{-57}\right)$ \\
\hline G & -0.18 & -0.47 & $\underline{-0.31}(<0.05)$ & $\underline{-0.25}(<0.01)$ & $\underline{-0.16}(<0.0001)$ \\
\hline C & -0.79 & $\underline{-0.71}(<0.05)$ & $\underline{-0.60}(<0.0001)$ & $\underline{-0.57}\left(<10^{-20}\right)$ & $\underline{-0.53}\left(<10^{-67}\right)$ \\
\hline A & 0.60 & $\underline{0.64}(<0.01)$ & $\underline{0.54}(<0.05)$ & $\underline{0.49}\left(<10^{-11}\right)$ & $\underline{0.28}\left(<10^{-15}\right)$ \\
\hline $\mathrm{T}$ & -0.13 & 0.33 & -0.02 & 0.00 & -0.04 \\
\hline
\end{tabular}

$\mathrm{X}$ indicates the position of the mutated nucleobase and $\mathrm{N}$ any base. The correlation coefficients that are statistically significant and for which the null hypothesis is rejected are underlined, with the $P$-values below $\alpha=0.05$ reported in parentheses. Note that the increase in sample size, from doublet to sextuplet motifs, also contributes to the increase of the statistical significance of the correlation

taking more flanking residues into account, to -0.3 for sextuplets, while becoming even more statistically significant. In other words, the lower the vIP of the sequence including the SBS, the higher the probability of the nucleotide substitution. Different biophysical mechanisms are likely to be responsible for this behavior, which will be discussed in the next section.

Interestingly, if we focus on the linear correlation between vIP and normalized SBS frequency separately for each of the four wild-type nucleobases, we see that the global anticorrelation is mainly due to the sequence motifs with a substituted guanine or cytosine. Motifs with cytosine substitutions, in particular, show the strongest anticorrelation with the vIP values, which even exceeds the correlation of guanine mutations, even though the latter base has the lowest vIP. Indeed, SBSs involving cytosine have linear correlations between -0.7 for triplets and -0.5 for sextuplets.

In contrast, motifs with a substituted adenine or thymine behave differently. They display no or even a positive linear correlation with their vIPs. More precisely, Ade-SBSs show a positive correlation, while Thy-SBSs have basically no correlation. This is true in all gene regions with a single exception, synonymous mutations in exons. The latter have a distinct behavior: substitutions involving thymine show a slight negative correlation with vIPs, while SBSs involving adenine substitutions do not correlate at all.

Let us have a closer look at Fig. 1 and Additional file 1: Figure S1-S4. Even though the linear correlation coefficients are statistically significant, it seems that the actual correlation is rather non-linear. This is confirmed by the higher values of the non-linear correlation coefficients obtained by fitting the data with a third-degree polynomial function (see Methods), which are reported in Additional file 1: Table S1. For low vIP values, the regression curve is strongly decreasing, while it is constant or slightly increases for large vIP values.

While the correlation coefficients between the vIP of the motifs and the normalized SBS frequencies show similar 

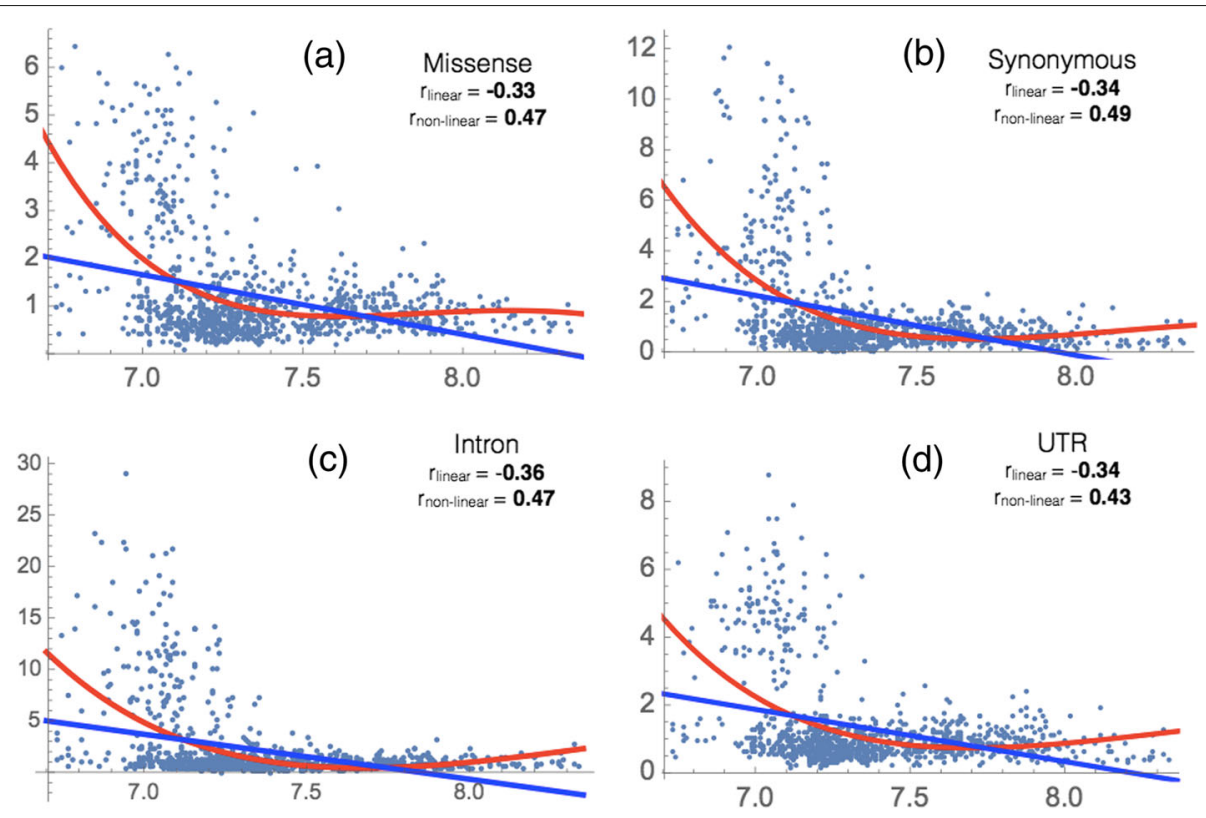

Fig. 1 Normalized frequency of observation of SBSs as a function of the vIP (in eV) of nucleobase quintuplets NNXNN, where X indicates the SBS and $\mathrm{N}$ any base, for missense, synonymous, intron and UTR mutations. Both the linear (blue) and polynomial (red) regression lines are drawn. The linear $\left(r_{\text {linear }}\right)$ and non-linear $\left(r_{\text {non-linear }}\right)$ correlation coefficients between the SBS frequencies and the vIP of the quintuplets are indicated

trends across the different gene regions, the regression slopes vary substantially. They are the lowest for missense exon mutations and UTR variants, about two times higher for synonymous exon mutations and about three times higher for mutations in introns, as seen in Table 4. This result is related to the observation that the normalized SBS frequencies have a much larger variance for synonymous and intron mutations than for missense and UTR substitutions (see Table 1). It means that, whereas the chance of having an SBS is larger for low-vIP motifs in all gene regions, this tendency is stronger for synonymous mutations and even more in introns. This leads us to conclude that different mutation mechanisms are probably involved in the different regions. We will come back to this point in the "Discussion" section.
In summary, the correlation between normalized SBS frequencies and vIP values is highly statistically significant and extends along the nucleobase stack up to sextuplets at least. This strongly supports the importance of the electronic properties of the DNA and the charge migration along the DNA stack in the modulation of the SBSs.

\section{$5^{\prime}-3^{\prime}$ asymmetry of the flanking sequence in the vIP-SBS frequency correlations}

We analyzed whether the base sequence flanking an SBS in the 5' direction is more, or less, informative than the sequence in the 3' direction. For that purpose, we computed the SBS frequency of the nucleotide sequences 5'-XN-3', 5'-XNN-3' and 5'-XNNN-3' and of the "inverse" sequences 5'-NX-3', 5'-NNX-3', 5'-NNNX-3'. We then

Table 4 Slope of the regression lines of the normalized SBS frequency as a function of the vIP of the corresponding wild-type nucleotide motifs in the different gene regions

\begin{tabular}{|c|c|c|c|c|c|}
\hline$x$ & DoubletsXN & Triplets $N X N$ & Quadruplets NXNN & Quintuplets NNXNN & SextupletsNNXNNN \\
\hline \multicolumn{6}{|c|}{ Missense mutations in exons } \\
\hline All & -0.6 & -1.1 & -1.1 & -1.3 & -1.5 \\
\hline \multicolumn{6}{|c|}{ Synonymous mutations in exons } \\
\hline All & -1.4 & -1.8 & -2.1 & -2.4 & -2.6 \\
\hline \multicolumn{6}{|c|}{ Mutations in introns } \\
\hline All & -1.8 & -3.7 & -3.7 & -4.4 & -5.3 \\
\hline \multicolumn{6}{|c|}{ Mutations in UTRs } \\
\hline All & -0.9 & -1.4 & -1.4 & -1.5 & -1.7 \\
\hline
\end{tabular}

$\mathrm{X}$ indicates the position of the mutated nucleobase and $\mathrm{N}$ any base 
Table 5 5'-3' directional asymmetry of the SBS flanking sequences, observed from differences in Pearson's linear correlation coefficients

\begin{tabular}{lllll}
\hline & Missense & Synonymous & Intron & UTR \\
\hline$r_{\text {XN }}-r_{\text {NX }}$ & -0.08 & -0.27 & -0.08 & -0.12 \\
$r_{\text {XNN }}-r_{\text {NNX }}$ & -0.19 & -0.23 & -0.14 & -0.17 \\
$\underline{r_{\text {XNNN }}-r_{\text {NNNX }}}$ & $\underline{-0.21}(<0.005)$ & $\underline{-0.14}(<0.05)$ & $\underline{-0.16}(<0.05)$ & $\underline{-0.16}(<0.05)$ \\
\hline
\end{tabular}

See legend of Table 3 for further details

computed the difference of the correlation coefficients $r_{\mathrm{XN} . \mathrm{N}}$ and $r_{\mathrm{N} . . \mathrm{NX}}$ between vIPs and normalized SBS frequencies. The results are given in Table 5.

Interestingly, we found a directional 5'-3' asymmetry: the linear correlation coefficients are always more negative when the flanking sequence is considered towards the 3' end rather than towards the 5' end. This effect is weak but statistically significant, with values in the -0.1 to -0.2 range, and seems to be independent of the gene region considered. Moreover, it extends beyond the dinucleotide level up to quadruplets at least.

This 5'-3' asymmetry can be put in relation with the preferential direction of the charge transport along the DNA base stacking, which appears to be from 5' to 3' [27].

\section{Connection between vIP and pathogenicity of the SBSs}

Electron hole transport can be expected to play an important role not only in the mutability of specific DNA sequences but also in the pathogenic effect of SBSs, since these substitutions can interfere, for example, with some repair mechanisms through the modification of the affinity for DNA-processing enzymes involved in DNA repair, or with basic cellular processes such as transcription and replication through the modification of targeted proteinDNA interactions.

The first quantity that we computed to gain insights into these matters is the linear correlation between the vIP of the motifs consisting of the wild-type bases and their flanking sequence and the difference in frequency $(\Delta v)$ between pathogenic and neutral substitutions normalized by the frequency of the motif in the considered gene region. We grouped for this purpose pathogenic and likely pathogenic annotations, as well as benign and likely benign annotations.

As seen in Table 6, there is a small but significant correlation between vIP values and $\Delta v$, especially for transversions and missense mutations. In introns and for synonymous mutations, the correlations are not statistically significant, but this is probably due to the small number of annotated SBSs of these types, which prevents us to draw robust conclusions. In summary, pathogenic missense
Table 6 Pearson's linear correlation coefficients between the vIP of the wild type base surrounded by its flanking sequence and the difference in normalized frequency $\Delta v$ between pathogenic and benign substitutions

\begin{tabular}{|c|c|c|c|}
\hline & DoubletsXN & Triplets NXN & Quadruplets NXNN \\
\hline \multicolumn{4}{|c|}{ Missense mutations in exons } \\
\hline All & -0.35 & -0.18 & $\underline{-0.15}(<0.001)$ \\
\hline Transitions & -0.32 & -0.10 & $\underline{-0.06}(<0.05)$ \\
\hline Transversions & -0.39 & $\underline{-0.35}(<0.0005))$ & $\underline{-0.27}\left(<10^{-6}\right)$ \\
\hline \multicolumn{4}{|c|}{ Synonymous mutations in exons } \\
\hline All & -0.41 & $\underline{-0.20}(<0.05)$ & -0.11 \\
\hline Transitions & -0.37 & -0.16 & -0.08 \\
\hline Transversions & -0.46 & -0.21 & -0.15 \\
\hline \multicolumn{4}{|c|}{ Mutations in introns } \\
\hline All & 0.20 & 0.17 & 0.06 \\
\hline Transitions & 0.00 & 0.27 & 0.08 \\
\hline Transversions & 0.10 & -0.09 & -0.02 \\
\hline
\end{tabular}

See legend of Table 3 for further details

mutations are enriched with low vIP sequences, while we cannot conclude for other types of SBSs at this stage.

We also computed the correlation between the absolute values of the change in vIP between the wild type and mutated nucleotides $(|\Delta \mathrm{vIP}|)$ and $\Delta v$. As we can see from Table 7 , the correlation is positive and quite high: from 0.5 in UTRs up to 0.7 for intron mutations, and missense and synonymous mutations in exons. This suggests that a larger change in the vIP of the nucleotide base at which the mutation occurs is associated to a higher chance of the mutation to be pathogenic.

However, when calculating the correlation between $\Delta v$ and $|\Delta v I P|$ by taking into account the flanking sequences, no statistically significant result was found. This absence of correlation has several possible explanations. It could be due to the limited accuracy of the vIP calculations and the fact that differences in vIPs have a much larger relative error than vIPs themselves. This is moreover supported by the fact that, when using the calculated vIPs rather than the experimental ones, the correlations shown in Table 7 drop down to about 0.40 . However, this result could also be taken to mean that the observed correlation between $\Delta v$ and $|\Delta v \mathrm{vIP}|$ is not due to charge migration, but rather to other biophysical phenomena.

\section{Discussion}

Our results show a clear overall anticorrelation between the vIP of short nucleotide sequences and the normalized frequencies of SBSs in these sequences. Note that the actual relation between vIPs and SBS frequencies appears to be non-linear (Fig. 1 and Additional file 1: Figure S1-S4), 
and we therefore also fitted the data using third-degree polynomials.

On the average, the lower the vIP of a nucleobase sequence motif, the more frequent the SBSs in this motif and vice versa. This effect extends far beyond the dinucleotide units - it is still clearly visible for base sextuplets.

These results support the fundamental role played by electron-hole transfer along the DNA stack in mutagenesis processes, which in some cases lead to deleterious phenotypes. The rationale behind this finding is that DNA charge transfer can interfere with different biomolecular mechanisms, triggering SBSs and/or preventing enzymatic-driven DNA repair mechanisms. Indeed, oxidative stress due to physical or chemical agents is likely to cause the extraction of an electron from DNA. Examples of such agents include exposure to ionizing radiation, long-wave ultraviolet light, and reactive oxygen species, just to mention some of them. The hole can then migrate and remain trapped in a minimum of the ionization potentials, and possibly trigger a base substitution. For example, guanine radical cations can react with water to form 8-oxo-guanine, which can trigger $\mathrm{G}: \mathrm{C} \rightarrow \mathrm{T}: \mathrm{A}$ transversions [28], while oxidative damage of the cytosine can undergo deamination and/or dehydration to a poorly repaired uracil, leading after replication to the $\mathrm{G}: \mathrm{C} \rightarrow \mathrm{A}: \mathrm{T}$ transition (for further information on oxidative damage of DNA bases, see [29]).

Furthermore, our analyses revealed a difference in tendency between Gua and Cyt substitutions on the one hand, and Ade and Thy SBSs on the other hand. For the former, a negative linear correlation between the vIP and the normalized frequency is observed, whereas for the latter the correlation coefficient is slightly positive or zero. Most interestingly, cytosine SBSs appear to be quite special: both their linear and non-linear correlations with vIPs are systematically better than for other bases, with linear and non-linear correlation coefficients of -0.7 and 0.9 for triplet SBSs. This is a very strong indication of the role that charge transport has in these type of substitutions.

Although the correlation coefficients between the vIP and the SBS frequency are very similar across the different gene regions, the slope of the linear regression lines varies. Indeed, the chance of having a base substitution in low-vIP sequences is much higher in introns than in

Table 7 Pearson's linear correlation coefficients between the absolute values of the vIP changes upon SBSs $(|\Delta v| P \mid)$ and the difference in normalized frequency $\Delta v$ between pathogenic and neutral substitutions

\begin{tabular}{llll}
\hline Missense & Synonymous & Intron & UTR \\
\hline$\underline{\underline{0.68}}(<0.05)$ & $\underline{0.73}(<0.005)$ & $\underline{0.67}(<0.05)$ & 0.49 \\
\hline \hline See legend of Table 3 for further details & &
\end{tabular}

exons and UTRs, and is also higher for synonymous than for missense substitutions. A priori, we did not expect differences in the mechanisms that trigger SBSs in the different gene regions, and certainly not between synonymous and missense substitutions. It can be argued that the latter result could be due to the lethality of some base substitutions that are thus not observed; such mutations are expected to be more often missense than synonymous, as the former modify the proteins and are thus more likely to be deleterious for their function. Or simply, the reason could be that there are more constraints on missense mutations due to their impact on the stability and function of the encoded proteins. Another possible explanation is that the synonymous codon selection is modulated by the constraint of maintaining specific electronic charge properties along the DNA.

Moreover, the larger slopes of the regression lines (in absolute value) observed for intron mutations compared to exon substitutions are related to the larger variance in their SBS frequencies (Table 1). This result can be put is relation with the observation that GGG triplets, which have the lowest vIP, are quite frequent near the $5^{\prime}$ termini of introns, where they have been suggested to serve as sacrificial anodes to protect the protein-coding part of the genes [30]. However, to definitely interpret our results, we need more insights into what occurs in the deep intronic regions, which mutagenesis mechanisms play a role, and how the correct functioning of the splicing process occurs [31].

Finally, we observed a difference between the linear correlation coefficients of SBSs occurring in 5'- and 3'UTRs, as seen in Additional file 1: Table S3. 5'-UTR substitutions appear to be similar to synonymous mutations and 3'-UTR substitutions to missense mutations. The role of vIP is likely to be important in UTRs but has still to be fully understood. Such regions act to regulate and modulate the protein gene expression at the posttranscriptional level. They contain regulatory sequences that impact the mRNA stability, transport and translational efficiency. Charge transport in these regions can influence a variety of factors and its modification can thus lead to an impact on the normal functioning of the protein synthesis.

Another interesting result is the asymmetry with respect to the flanking sequence when computing the correlations between vIPs and SBS rates. Indeed, the correlation is always better when the mutated base is near the $5^{\prime}$ end of the considered sequence segment rather than near the 3' terminus. This directional dependence could reflect the fact that the physical structure of the DNA stack is asymmetric. In particular, the overlap between successive bases differs between 5'-AB-3' and 5'-BA-3' configurations [32]. This leads in turn to nucleobase stack vIPs which are not symmetric with respect to sequence 
inversions, and thus to a DNA charge transport with a directional preference. Indeed, even though it remains difficult to test experimentally, electron hole transfer seems to be more efficient in the 5'-3' than in the 3'-5' direction [27]. Hence, if we assume that the charge transport mechanisms are strictly connected to mutagenesis processes, the vIP of the flanking sequence in the 3' direction after the substituted nucleobase is expected to be better correlated with the observed SBS frequency. This corresponds indeed to what we observe.

Not only mutability appears to be related with electronic properties of DNA, but also pathogenicity. Indeed, we found a weak but statistically significant correlation between the pathogenicity of the SBSs and the vIP values of the wild-type bases and their flanking sequences. It is prevalently observed for missense mutations, but it has to be noted that the number of disease annotations for other types of variants is too low to yield reliable statistics. Note that this correlation is always larger for transversions than for transitions, and that the former are also more pathogenic in general.

Finally, we found quite a high correlation between the change in vIP between the wild-type and mutant nucleobases in absolute value $(|\Delta \mathrm{vIP}|)$ and the pathogenicity of the SBSs. However, the correlation disappears when taking also into account the flanking sequences. This can be taken to mean that it is not the vIP that drives the correlation, but other characteristics of the four nucleobases. But it can also be due to the approximations done when calculating vIPs, which are described in the last Methods subsection. Indeed, differences in vIP values are tiny, and inaccuracies in vIPs have therefore a larger effect on $\Delta v$ IPs than on the vIPs themselves. More analyses are needed to settle this interesting issue.

Our results thus suggest that vIPs and the associated DNA charge transfer could be not only related to the mutation rate, but also to the pathogenicity of the mutations. This effect can be attributed to the modification of biophysical mechanisms such as DNA-protein binding, or base or nucleotide excision repair mechanisms [33, 34].

\section{Conclusion}

Since the last two decades, it is becoming increasingly clear that DNA charge transport has a fundamental role in a wide range of biomolecular processes. Sometimes, cells use it for long-range biological sensing and signaling and it is then of vital importance, while in other cases oxidative stress induces electron holes that migrate along the base stack and can, for example, lead to single base mismatches via a wide series of chemical mechanisms, or interfere with the binding of specific proteins.

In this study we focused on the analysis of the SBS rates, and found a clear non-linear correlation with the vIP values of the wild-type bases and their neighboring sequence. This correlation differs for substitutions of Gua and Cyt bases and for Ade and Thy, and the slope of the regression line varies between exons and introns, and between synonymous and missense mutations. A 5'3' directional asymmetry of the correlation is another indication of the importance of DNA charge transfer in the mutagenesis mechanisms. We also found a weak relation between the difference in frequency of pathogenic and neutral mutations and the vIP values, observing that pathogenic mutations tend to be embedded in base sequences of lower vIP. A detailed understanding of these results is currently out of reach, but we guess that it would constitute an important step toward the understanding of DNA mutability and how mutations cause pathogenic phenotypes.

Several points remain to be clarified and further explored to get a clearer picture. Our first plan is to extend our analysis to longer base sequences, in view of understanding up to which point there is a signal between SBS frequencies and vIP values. Indeed, even though a electron hole trapped in a potential well remains localized in a region composed of 2-4 nucleobases, it can migrate much further away, over distances up to $200 \AA$ [16]. The calculation of the vIP of longer nucleobase stacks is increasingly computationally demanding, but cannot be avoided. As a test, we estimated the vIP of base sequences in two ways, either by calculating directly the vIP of the whole segment using MP2, or by estimating it as the average of the vIPs of the single nucleobases that compose it. We found that SBS frequencies correlate better with the vIP values obtained in the first manner, as shown in Additional file 2: Table S4. The stacked base structure has thus definitely to be considered if we want to explore the behavior of the charge transfer at the quantum level.

vIP values are known to change as a function of the DNA structure [22], so that trapped holes can start migrating again upon (even small) DNA conformational modifications. In this study, we considered only DNA segments in standard B-conformations, but our analysis can easily be extended to A-conformations and other conformations observed in experimental structures. It would also be worthwhile to take into account different nucleotide modifications, such as the 5-methylation of cytosines, as they obviously impact on the vIP values and thus on the derivation of our results.

Another perspective consists in focusing on SBSs occurring in some specific diseases such as cancer and to analyze the link between the observed mutational signatures and vIP values. In particular, the extension of our investigation to datasets such as COSMIC [35], in which somatic cancer mutations are collected, could certainly help to get more insights into the role of the vIP in the mutation processes. It will be interesting to connect our findings to the 30 mutational signatures that have been 
observed [35] and are unique combinations of mutations occurring across the spectrum of human cancer types. Moreover, since such variants are not subject to selective pressure, which can be suspected to influence the correlations between the vIP values and the frequency of the DNA base motifs, we could expect even stronger signals compared to those observed in the present analysis.

All the analyses proposed here aim to better understand the mutagenesis processes and how oxidative stress caused by chemical and physical agents affect the electron-hole transfer in DNA and lead to nucleobase substitutions and pathogenic phenotypes.

\section{Methods}

\section{Dataset of mutations}

The SBS data were extracted from the Single Nucleotide Polymorphism Database (dbSNP) [36]. We focused on variants in gene regions, and for all of them, we collected the wild type and mutant nucleotides, the thirty flanking nucleotides, i.e. fifteen in the 5 ' direction and fifteen in the 3' direction, the region of the gene in which the mutation is inserted (exon, intron, 5'-UTR, or 3'UTR). If the variant is in an exon region, we moreover identified the exact position of the mutated site with respect to the translated codon in order to determine whether it corresponds to a missense or a synonymous mutation.

Our final SBS dataset is defined as the subset of these SBSs for which the clinical phenotype (benign, likely benign, likely pathogenic, pathogenic, variant of unclear significance, ...) is annotated in the ClinVar database [37].

Three quarters of the 190,000 variants from our SBS dataset occur in exon regions, while the remaining are variants in introns (about 25,000) and in UTR regions (30,000 in 3'-UTR and 8,000 in 5'-UTR). Among the exon SBSs, $72 \%$ are missense variants and $28 \%$ synonymous. Only one third of the variants have an established phenotypic effect (benign, likely benign, likely pathogenic, pathogenic), while the remaining two thirds of the variants have uncertain significance or other annotations.

\section{Frequency of nucleobase motifs}

We computed the normalized frequency of base pairs, doublets, triplets, up to sextuplets by dividing the frequency of their occurrence in the SBS dataset by their frequency of observation in each specific gene region (exon, intron, UTR). To estimate the latter frequencies, we used the UCSC Genome browser [38]. We first retrieved the positional table of the selected regions in the genome assembly GRCh38.p12, and then dowloaded the corresponding sequences from which we computed all the motif frequencies.

\section{Vertical ionization potentials of nucleobase sequences}

For computing the vIP of nucleobase sequences, we used the procedure set up in [22]. In a first stage, the four nucleobases Ade, Cyt, Gua, and Thy were considered separately. Their sugar cycle and phosphate group were omitted, and the glycosidic bond was replaced by a hydrogen atom. Their initial geometries were taken from the molecular-modeling program package Insight 2000 (Accelrys Inc.). These geometries were then optimized at second-order Møller-Plesset perturbation theory (MP2) [39] using the split-valence basis set 6-31G* with added d polarization functions on non-hydrogen atoms [40]. The Gaussian 09 program suite [41] was used for these, and all subsequent, quantum chemistry calculations.

The energy in gas phase of these optimized geometries was computed for the neutral species and for the radical cationic species, with one missing electron. The calculations for cationic molecules were performed with restricted open-shell procedures to prevent spin contamination problems. The vIP was defined as the difference between the energy of the cationic and neutral species.

In a second stage, we considered all possible singlestranded nucleobase stacks in standard B-conformation containing two, three or four nucleobases. The geometries of the stacks were taken from early fiber X-ray diffraction studies [42], using Insight 2000 (Accelrys Inc.). The isolated nucleobases, individually optimized at the MP2/6$31 G^{*}$ level, were superimposed onto the original bases forming the stacks, so as to minimize their root mean square deviation of atomic positions using the U3BEST algorithm [43]. The gas phase energies of these stacks of optimized nucleobases, for the neutral and radical cationic species, were calculated at MP2/6-31G* level, using the same geometry for both species. The vIP was computed as the difference between the energy of the radical cationic and neutral species, both adopting the same geometry.

Note that we used MP2 to calculate the vIPs rather than the hybrid density functional theory method M06-2X [44] that we used in [22]. Indeed, although the vIPs of the single nucleobases are closer to the experimental values when using M06-2X, the results on nucleobase stacks appear to be better when using MP2, as already described before [45]. For example, the GGG triplets have, as expected, the lowest vIP of all triplets with MP2, but not with M06-2X.

\section{Approximations in the VIP calculations}

We made several approximations when computing the vIPs of the nucleobase stacks. First, we omitted the sugarphosphate backbone. Of course, their inclusion would require too much computer time and memory, but there are other justifications. Calculations on Cyt and Thy indicated that the vIP values change according to the presence or absence of the sugar and phosphate moieties in 
gas phase, but much less in an aqueous solvent due to screening effects [46]. Moreover, $a b$ initio calculations and experimental data showed that the lowest ionization pathway comes from the nucleobase stacks and not from the sugar-phosphate backbone [46, 47].

The second approximation we made is to perform all calculations in gas phase. We made this choice to reduce computer time, but also because such calculations seem to be suitable for comparing the vIP values of various nucleobase stack sequences. Indeed, calculations on individual nucleobases highlighted the effect of the solvent in lowering the vIP values while maintaining the relative ordering between the bases $[48,49]$. Moreover, dropping both the sugar-phosphate backbone and the solvent have opposite effects that tend to cancel out [46].

We also calculated vIPs of single-stranded rather than double-stranded nucleobase stacks. This is justified by the fact that electron holes migrate along a single strand and jump only rarely to the complementary strand [50]. Moreover, we assumed a fixed B-conformation, although DNA molecules have some flexibility and the vIPs of nucleobase stacks in A- and B-conformations have been shown to differ significantly [22].

\section{Correlation coefficients and statistical tests}

When we refer to the linear correlation coefficient of two variables $X$ and $Y$, we mean the Pearson correlation coefficient. We also defined a non-linear correlation coefficient, obtained using a polynomial function of third degree:

$$
f(X)=a+b X^{-1}+c X^{-2}+d X^{-3}
$$

where the parameters $a, b, c$, and $d$ are identified so as to minimize the root mean square deviation between $f(X)$ and $Y$. The non-linear correlation coefficient is the Pearson correlation coefficient computed between $f(X)$ and $Y$.

To check the statistical significance of correlations or pairs of correlations, we performed a hypothesis test on the bivariate sample against the null hypothesis. This was done by applying the Fischer z-transformation to the sample, followed by a z-test.

\section{Additional files}

Additional file 1: Table S1. Non-linear correlation coefficients between the vIP of the wild-type nucleotide motifs and the normalized SBS frequency in the different gene regions. Table S2. Statistical power and confidence interval for the linear correlation coefficients between the vIPs of the wild-type nucleotide motifs and the normalized SBS frequencies. Table S3. Pearson's linear correlation coefficients between the vIP of the wild-type nucleotides and their flanking base sequences and the normalized SBS frequency in UTR-5 and UTR-3 regions. Figure S1-S4. Normalized frequency of observation of missense, synonymous, intron and UTR SBSS as a function of the VIP (in eV) of nucleobase quintuplets NNXNN, where $X$ indicates the SBS and $N$ any base. (PDF $985 \mathrm{~kb}$ )
Additional file 2: Table S4. Excel file with the normalized and the standard SBS frequencies for single bases (sheet-Single), base doublets (sheet-Doublet), triplets (sheet-Triplet), quadruplets (sheet-Quadruplet), and quintuplets (sheet-Quintuplet). In (sheet-ObsExp), the frequency of observation of the motifs in the different gene regions are reported. (sheet-AverageVSComp) contains the comparison between the vIPs of the quadruplets computed using MP2 with those computed by averaging the MP2 VIPs of the overlapping triplets or doublets, and of the constituent nucleobases. Finally, in (sheet-MP2vsM06-2X), the comparison of the triplet VIPs computed with MP2 with those computed with M06-2X is reported. (XLSX $330 \mathrm{~kb})$

\section{Abbreviations}

mC: Methylated cytosine; MP2: Møller-Plesset perturbation theory; SBSs: Single base substitutions; UTR: Untranslated region; vIP: Vertical ionization potential

\section{Acknowledgments}

We thank Jonathan Velu for help with the SBS dataset construction and Emilie Cauët for interesting discussions.

\section{About this supplement}

This article has been published as part of BMC Genomics Volume 20 Supplement 8, 2019: Proceedings of Varl-COS1 2018: identification and annotation of genetic variants in the context of structure, function, and disease: genomics. The full contents of the supplement are available online at https://bmcgenomics. biomedcentral.com/articles/supplements/volume-20-supplement-8.

\section{Authors' contributions}

FP and MR designed and performed this study, interpreted the results and wrote the manuscript. Both authors read and approved the final manuscript

\section{Funding}

Publication costs for this article were provided by the Fund for Scientific Research - FNRS through a PDR research project. FP and MR are FNRS postdoctoral researcher and research director, respectively.

\section{Availability of data and materials}

The full contents of the supplementary information are available online at https://bmcgenomics.biomedcentral.com/

\section{Ethics approval and consent to participate}

\section{Not required.}

\section{Consent for publication}

Not applicable.

\section{Competing interests}

The authors declare that they have no competing interests.

\section{Author details}

${ }^{1}$ Computational Biology and Bioinformatics, Université Libre de Bruxelles, Roosevelt Ave. 50 1050, Bruxelles, Belgium. ${ }^{2}$ John von Neumann Institute for Computing, Jülich Supercomputer Centre, Forschungszentrum Jülich, 52428 Jülich, Germany.

\section{Published: 16 July 2019}

\section{References}

1. Alexandrov LB. Signatures of mutational processes in human cancer. Nature. 2013;500:415-21.

2. Bacolla A, Cooper DN, Vasquez KM. Mechanisms of base substitution mutagenesis in cancer genomes. Genes. 2014;5:108-46.

3. Cooper DN1 MortM Stenson PD. Methylation-mediated deamination of 5-methylcytosine appears to give rise to mutations causing human inherited disease in CpNpG trinucleotides, as well as in CpG dinucleotides. Hum Genomics. 2010;4:406-10.

4. Cooper DN, Youssoufian H. The CpG dinucleotide and human genetic disease. Hum Genet. 1988;78:151-5.

5. Alexandrov LB, Stratton MR. Mutational signatures: the patterns of somatic mutations hidden in cancer genomes. Curr Opin Genet Dev. 2014;24:52-60. 
6. Harris RS, Petersen-Mahrt SK, Neuberger MS. RNA editing enzyme APOBEC1 and some of its homologs can act as DNA mutators. Mol Cell. 2002:10:1247-53.

7. Alexandrov LB, Jones PH, Wedge DC, Sale JE, Campbell PJ, Nik-Zainal S, Stratton MR. Clock-like mutational processes in human somatic cells. Nat Genet. 2015;47:1402-07.

8. Nik-Zainal $\mathrm{S}$, et al. Landscape of somatic mutations in 560 breast cancer whole-genome sequences. Nature. 2016;534:47-54.

9. India Project Team of the International Cancer Genome Consortium. Mutational landscape of gingivo-buccal oral squamous cell carcinoma reveals new recurrently-mutated genes and molecular subgroups. Nat Commun. 4;2013:2873.

10. Ma L, Zhang T, Huang Z, Jiang $X$, Tao S. Patterns of nucleotides that flank substitutions in human orthologous genes. BMC Genom. 2010;11:416.

11. Giese B. Long-distance electron transfer through DNA. Annu Rev Biochem. 2002;71:51-70.

12. Suarez-Villagran MY, Miller JH. Computational DNA hole spectroscopy: A new tool to predict mutation hotspots, critical base pairs, and disease driver mutations. Sci Rep. 2015;5:13571.

13. Suarez-Villagran MY, Azevedo RBR, Miller JH. Influence of Electron-Holes on DNA Sequence-Specific Mutation Rates. Genome Biol Evol. 2018;10: 1039-47.

14. Boal AK, Genereux JC, Sontz PA, Gralnick JA, Newman DK, Barton JK, Redox signaling between DNA. repair proteins for efficient lesion detection. Proc Natl Acad Sci USA. 2009;106:15237-42.

15. Aguirre J, Ríos-Momberg M, Hewitt D, Hansberg W. Reactive oxygen species and development in microbial eukaryotes. Trends Microbiol. 2005;13:111-8.

16. Genereux J, Boal A, Barton J. DNA-mediated charge transport in redox sensing and signaling. J Am Chem Soc. 2010;132:891-905.

17. Rooman M, Cauët E, Liévin J, Wintjens R. Conformations consistent with charge migration observed in DNA and RNA X-ray structures. J Biomol Struct Dyn. 2011;28:949-53.

18. Bacolla A, Temiz NA, Yi M, Ivanic J, Cer RZ, Donohue DE, Ball EV, Mudunuri US, Wang G, Jain A, Volfovsky N, Luke BT, Stephens RM, Cooper DN, Collins JR, Vasquez KM. Guanine holes are prominent targets for mutation in cancer and inherited disease. PLoS Genet. 2013;9: e1003816.

19. Shih CT, Wells SA, Hsu CL, Cheng YY, Römer RA. The interplay of mutations and electronic properties in disease-related genes. Sci Rep. 2012;2:272.

20. Arnold AR, Grodick MA, Barton JK. DNA Charge Transport: from Chemical Principles to the Cell. Cell Chem Biol. 2016;23:183-97.

21. Zwang TJ, Tse ECM, Barton JK. Sensing DNA through DNA Charge Transport. ACS Chem Biol. 2018;13:1799-809.

22. Rooman $M$, Wintjens $R$. Sequence and conformation effects on ionization potential and charge distribution of homo-nucleobase stacks using M06-2X hybrid density functional theory calculations. J Biomol Struct Dyn. 2014;32:532-45.

23. Triberis GP, Dimakogianni M. DNA in the material world: electrical properties and nano-applications. Recent Pat Nanotechnol. 2009;3: 135-53.

24. Duret L, Galtier N. The covariation between TpA deficiency, CpG deficiency, and $\mathrm{G}+\mathrm{C}$ content of human isochores is due to a mathematical artifact. Mol Biol Evol. 2000;17:1620-5.

25. Zhang Z, Gerstein M. Patterns of nucleotide substitution, insertion and deletion in the human genome inferred from pseudogenes. Nucleic Acids Res. 2003;31:5338-48.

26. Nachman MW, Crowell SL. Estimate of the mutation rate per nucleotide in humans. Genetics. 2000;156:297-304.

27. O'Neill MA, Barton JK. Effects of strand and directional asymmetry on base-base coupling and charge transfer in double-helical DNA. PNAS. 2002;99:16543-50.

28. Kino K, Sugiyama $\mathrm{H}$. Possible cause of $\mathrm{G}: \mathrm{C} \rightarrow \mathrm{C}: \mathrm{G}$ transversion mutation by guanine oxidation product, imidazolone. Chem Biol. 2001;8:369-78.

29. Cadet J, Wagner JR. DNA Base Damage by Reactive Oxygen Species, Oxidizing Agents, and UV Radiation. Cold Spring Harb Perspect Biol. 2013;5:a012559.

30. Friedman KA, Heller A. On the Non-Uniform Distribution of Guanine in Introns of Human Genes:? Possible Protection of Exons against Oxidation by Proximal Intron Poly-G Sequences. J Phys Chem B. 2001;105:11859-65.
31. Vaz-Drago R, Custodio N, Carmo-Fonseca M. Deep intronic mutations and human disease. Human Genet. 2017;136:1093-111.

32. In: Wagenknecht HA, Gray HB, editors. Charge Transfer in DNA: From Mechanism to Application. VCH Verlag GmbH \& Co: Wiley; 2005.

33. Schärer OD. Nucleotide Excision Repair in Eukaryotes. Cold Spring Harb Perspect Biol. 2013:5:a012609.

34. Krokan HE, Bjoras M. Base Excision Repair. Cold Spring Harb Perspect Biol. 2013;5:a012583.

35. Forbes AS, et al. COSMIC: somatic cancer genetics at high-resolution. Nucleic Acids Res. 2017;45:D777-83.

36. Sherry ST, Ward MH, Kholodov M, Baker J, Phan L, Smigielski EM, Sirotkin K. dbSNP: the NCBI database of genetic variation. Nucleic Acids Res. 2001;29:308-11.

37. Landrum MJ, Lee JM, Riley GR, Jang W, Rubinstein WS, Church DM, Maglott DR. ClinVar: public archive of relationships among sequence variation and human phenotype. Nucleic Acids Res. 2014;42:D980-5.

38. Table Browser UCSC, Karolchik D, Hinrichs AS, Furey TS, Roskin KM, Sugnet CW, Haussler D, Kent WJ. The UCSC Table Browser data retrieval tool. Nucleic Acids Res. 2004;32:D493-6.

39. Möller C, Plesset MS. Note on an approximation treatment for many-electron systems. Phys Rev. 1934;46:618-22.

40. Hariharan PC, Pople JA. Influence of polarization functions on MO hydrogenation energies. Theoretica Chimica Acta. 1973;28:213-22.

41. Frisch MJ, et al. Revision A.02. Wallingford CT: Gaussian, Inc; 2016.

42. Arnott S, Hukins DWL. Optimised parameters for A-DNA and B-DNA. Biochem Biophys Res Commun. 1972;47:1504-9.

43. Kabsch W. A solution for the best rotation to relate two sets of vectors. Acta Crystallogr A. 1976;32:922-3.

44. Zhao Y, Truhlar DG. The M06 suite of density functional for main group thermochemistry, thermochemical kinetics, noncovalent interactions, excited states, a, d transition elements: Two new functional and systematic testing of four M06-class functional and 12 other functional. Theor Chem Accounts. 2008;120:215-41.

45. Valdes H, Pluháčková K, Pitonák M, Řezáča J, Hobza P. Benchmark database on isolated small peptides containing an aromatic side chain: comparison between wave function and density functional theory methods and empirical force field. Phys Chem Chem Phys. 2008;10: 2747-57.

46. Slaviček $P$, Winter $B$, Faubel M, Bradforth $S$, Jungwirth $P$. Ionization energies of aqueous nucleic acids: Photoelectron spectroscopy of pyrimidine nucleosides and ab initio calculations. J Am Chem Soc. 2009;131:6460-7.

47. Fernando H, Papadantonakis GA, Kim NS, LeBreton PR. Conduction-band-edge ionization thresholds of DNA components in aqueous solution. Proc Natl Acad Sci USA. 1998;95:5550-5.

48. Cauët $\mathrm{E}$, Valiev M, Weare JH. Vertical ionization potentials of nucleobases in a fully solvated DNA environment. J Phys Chem B. 2010;114:5886-94.

49. Close DM. Calculation of the ionization potentials of the DNA bases in aqueous medium. J Phys Chem A. 2004;108:10376-9.

50. O'Neill P, Parker AW, Plumb MA. Siebbeles LDA. Guanine Modifications Following lonization of DNA Occurs Predominantly via Intra- and Not Interstrand Charge Migration: An Experimental and Theoretical Study. J Phys Chem B. 2001;105:5283-90.

\section{Publisher's Note}

Springer Nature remains neutral with regard to jurisdictional claims in published maps and institutional affiliations. 